\title{
Constraints of the KP Hierarchy and Multilinear Forms
}

\author{
Yi Cheng', Walter Strampp ${ }^{2}$, Bin Zhang ${ }^{1}$ \\ ${ }^{1}$ Department of Mathematics, University of Science and Technology of China, Hefei. Anhui \\ 230026, People's Republic of China \\ ${ }^{2}$ Fachbereich 17-Mathematik/ Informatik, Universität-GH Kassel, Holländische Str. 36, D-34109 \\ Kassel, Germany
}

Received: 6 December 1993/in revised form: 20 May 1994

\begin{abstract}
We consider the trilinear form of the Kaup-Broer system which gives rise to solutions in Wronskian form. The Kaup-Broer system is connected with AKNS system through a gauge transformation. The AKNS hierarchy can be understood as a generalized 1-constraint of the KP hierarchy. Imposing that constraint on Sato's equation we obtain the basic trilinear form and moreover a hierarchy of trilinear equations governing the AKNS flows. Similarly, hierarchies of multilinear forms are derived in the case of generalized k-constraints.
\end{abstract}

\section{Introduction}

\subsection{The Trilinear Form of the Kaup-Broer System.}

The Kaup-Broer system [4,5]

$$
\begin{aligned}
& h_{t_{2}}=\left(h_{x}+2 \chi^{*} h\right)_{x}, \\
& \chi_{t_{2}}^{*}=\left(-\chi_{x}^{*}+\chi^{* 2}+2 h\right)_{x},
\end{aligned}
$$

plays an important role in the theory of nonlinear water waves. It has been discussed in [6] where its Lax form and tri-Hamiltonian structure was established. The following ansatz for obtaining solutions has been made in [7],

$$
\begin{aligned}
h & =(\log \tau)_{x x}, \\
h \chi^{*} & =\frac{1}{2}\left((\log \tau)_{x t_{2}}-(\log \tau)_{x x x}\right) .
\end{aligned}
$$

This ansatz was motivated by the fact that Eq. (1.1) is nothing but the simplest equation of the KP hierarchy so it is already satisfied by (1.3), (1.4). In deriving 
an equation for the quantity $h \chi^{*}$ using (1.1), (1.2), we are lead to the trilinear form

$$
\left|\begin{array}{lll}
p_{0}^{+} p_{0}^{-}(\tau) & p_{0}^{+} p_{1}^{-}(\tau) & p_{0}^{+} p_{2}^{-}(\tau) \\
p_{1}^{+} p_{0}^{-}(\tau) & p_{1}^{+} p_{1}^{-}(\tau) & p_{1}^{+} p_{2}^{-}(\tau) \\
p_{2}^{+} p_{0}^{-}(\tau) & p_{2}^{+} p_{1}^{-}(\tau) & p_{2}^{+} p_{2}^{-}(\tau)
\end{array}\right|=0
$$

where we have employed the linear differential operators

$$
p_{l}^{+}=p_{l}(+\tilde{\partial}), \quad p_{l}^{-}=p_{l}(-\tilde{\partial}), \quad \tilde{\partial}=\left(\partial_{x}, \frac{1}{2} \partial_{t_{2}}, \frac{1}{3} \partial_{t_{3}}, \ldots\right)
$$

with the Schur polynomials $p_{i}(t), t=\left(t_{1}, t_{2}, t_{3}, \ldots\right)$, defined through

$$
e^{\xi(t, \lambda)}=\sum_{i=0}^{\infty} p_{i}(t) \lambda^{i}, \quad \xi(t, \lambda)=\sum_{i=1}^{\infty} t_{i} \lambda^{i}, \quad t_{1}=x .
$$

Then it has been shown [7-9] that the trilinear equation possesses solutions in Wronskian form

$$
\tau=\left|\begin{array}{cccc}
f & f_{x} & \cdots & f_{(N-1) x} \\
f_{x} & f_{x x} & \cdots & f_{N x} \\
\vdots & \vdots & \vdots & \vdots \\
f_{(N-1) x} & f_{N x} & \cdots & f_{(2 N-2) x}
\end{array}\right|,
$$

where

$$
f_{t_{2}}=f_{x x}
$$

Solutions of the trilinear equation (1.5) with interesting physical properties have been studied in [10].

\subsection{Generalized $k$-Constraints}

The AKNS system

$$
\begin{aligned}
& q_{t_{2}}=q_{x x}+2 q^{2} r \\
& r_{t_{2}}=-r_{x x}-2 q r^{2}
\end{aligned}
$$

transforms to the Kaup-Broer system through

$$
\begin{gathered}
h=q r, \\
\chi^{*}=-\frac{r_{x}}{r} .
\end{gathered}
$$

(Note that in terms of pseudodifferential operators this transformation corresponds to a gauge transformation between the KP and modified KP hierarchy [19].) The AKNS hierarchy appears as a subsystem of the KP hierarchy under generalized 1-constraint [11-20]. 
Let us briefly discuss generalized k-constraints. The KP hierarchy is given in Lax form by

$$
\partial_{t_{n}} L=\left[L, L_{-}^{n}\right] \text {. }
$$

All KP flows, i.e. the vector fields $\partial_{t_{n}}$ defined by (1.14) commute

$$
\left[\partial_{t_{n}}, \partial_{t_{m}}\right]=0 \text {. }
$$

There is another vector field commuting with $\partial_{t_{n}}$ which is sometimes called a ghost symmetry. Consider a vector field $\partial_{t_{\alpha}}$ acting on $L$ through

$$
\partial_{t_{\alpha}} L=\left[L, \psi \partial^{-1} \psi^{*}\right]
$$

where $\psi$ is the wave-function and $\psi^{*}$ the adjoint wave-function. Then we can show [20]

$$
\left[\partial_{t_{n}}, \partial_{t_{\alpha}}\right]=0
$$

Now, comparing (1.14) and (1.16) we introduce the so-called generalized kconstraint [11-20],

$$
\left(L^{k}\right)_{-}=q \partial^{-1} r .
$$

Indeed, it is well-known that this constraint which generalizes k-reduction

$$
\left(L^{k}\right)_{-}=0
$$

is compatible with the KP hierarchy. The generalized k-constraint leads to $1+1-$ dimensional integrable hierarchies

$$
\begin{aligned}
\left(L^{k}\right)_{t_{n}} & =\left[L^{k}, L_{-}^{n}\right], \\
q_{t_{n}} & =\left(L^{n}\right)_{+} q, \\
r_{t_{n}} & =-\left(L^{* n}\right)_{+} r,
\end{aligned}
$$

such as the AKNS $(\mathrm{k}=1)$, Yajima-Oikawa $(\mathrm{k}=2)$ or coupled Boussinesq-type $(\mathrm{k}=3)$ hierarchies [11-20]. $\left(L^{*}\right.$ is the adjoint of $\left.L\right)$.

\subsection{Multilinear Forms}

After obtaining the trilinear form (1.5) in [7], it was conjectured that the more general form

$$
\left|\begin{array}{lll}
p_{j 1}^{+} p_{i 1}^{-}(\tau) & p_{j 1}^{+} p_{i 2}^{-}(\tau) & p_{j 1}^{+} p_{i 3}^{-}(\tau) \\
p_{j 2}^{+} p_{l 1}^{-}(\tau) & p_{j 2}^{+} p_{i 2}^{-}(\tau) & p_{j 2}^{+} p_{i 3}^{-}(\tau) \\
p_{j 3}^{+} p_{i 1}^{-}(\tau) & p_{j 3}^{+} p_{i 2}^{-}(\tau) & p_{j 3}^{+} p_{l 3}^{-}(\tau)
\end{array}\right|=0
$$

may be related with higher Kaup-Broer flows. This conjecture is the subject of the present paper.

In Sect. 2 and 3 we shall gather a few notations from Sato's theory [1-3] and derive some useful formulas for dealing with constraints. In Sect. 4 the effect of k-constraints on Sato's equations will be described by a basic formula. In Sect. 5 
we shall show that the $2 \times 2$ determinant equations

$$
\left|\begin{array}{cc}
p_{1}^{+}\left(\omega_{i}\right) & p_{1}^{+}\left(\omega_{j}\right) \\
p_{n}^{+}\left(\omega_{i}\right) & p_{n}^{+}\left(\omega_{j}\right)
\end{array}\right|=0,
$$

where

$$
\omega_{j}=\frac{1}{\tau} p_{j}^{-}(\tau),
$$

describe the 1-constrained KP flows in terms of the KP $\tau$-function. In Sect. 6 we state that (under some formal assumptions) the set of Eqs. (1.23) appears as a consequence from the set of Eqs. (1.24).

If we consider the $2 \times 2$-determinants (1.24) with the lowest indices $i=1, j=2$ we obtain a hierarchy of flows where the $\mathrm{n}^{\text {th }}$ flow involves the time variables $t_{2}, t_{3}, \ldots, t_{n}$. In the final two sections we are concerned with the question how this pattern can be generalized to k-constraints. Similar to the 1-constraint we shall obtain multilinear forms for the k-constrained KP flows in terms of the KP $\tau$ function. Those multilinear forms take the shape of determinant equations, however the $2 \times 2$ pattern of the 1-constraint turns out to be exceptional. It seems that the multilinear forms for k-constraints with the lowest possible indices can be arranged as $(k+l) \times(k+l)$-determinants in the following diagram:

\begin{tabular}{c|c|c|c|c|c} 
constraints & flows & flows & flows & flows & $\ldots$ \\
\hline$k=1$ & $2 \times 2$ & $2 \times 2$ & $2 \times 2$ & $2 \times 2$ & $\ldots$ \\
$k=2$ & & $3 \times 3$ & $5 \times 5$ & $7 \times 7$ & $\ldots$ \\
$k=3$ & & & $4 \times 4$ & $6 \times 6$ & $\ldots$ \\
$k=4$ & & & & $5 \times 5$ & $\ldots$ \\
$\vdots$ & & & & & $\ldots$
\end{tabular}

The first row as well as the first two diagonals of this diagram are established in Sect. 7 and 8 .

\section{The Linear Problems in Sato's Theory}

With the pseudodifferential operator

$$
L=\partial_{x}+u_{2} \partial_{x}^{-1}+u_{3} \partial_{x}^{-2}+\cdots,
$$

we built up linear problems

$$
L \psi=\lambda \psi, \quad \psi_{t_{n}}=\left(L^{n}\right)_{+} \psi,
$$

which possess a formal solution (wave-functions)

$$
\psi(t, \lambda)=W e^{\xi(t, \hat{\imath})}=\widehat{W} e^{\xi(t, \hat{\imath})} .
$$

The pseudodifferential operator

$$
W=1+\omega_{1} \partial_{x}^{-1}+\omega_{2} \partial_{x}^{-2}+\cdots,
$$


is related to $L$ through $L=W \partial W^{-1}$ and $\widehat{W}$ is a formal series

$$
\widehat{W}=1+\omega_{1} \lambda^{-1}+\omega_{2} \lambda^{-2}+\cdots
$$

From the expression of the function $\omega_{j}$ in terms of the $\tau$-function through (1.25) we obtain that of the wave function

$$
\psi=\frac{\tau(t-\varepsilon(\lambda))}{\tau(t)} e^{\xi(t, \hat{\lambda}))}
$$

where we have used Taylor expansion for a function $f$

$$
f(t-\varepsilon(\lambda))=e^{\xi(-\tilde{c}, \lambda)}(f(t))=\sum_{n=0}^{\infty} p_{n}^{-}(f(t)) \lambda^{-n}
$$

with $\varepsilon(\lambda)=\left(1 / \lambda, 1 / 2 \lambda^{2}, 1 / 3 \lambda^{3}, \ldots\right)$, see [1-3]. $(2.2)$,

In [3] M. Sato and Y.Sato gave an equivalent formulation of the linear problems

$$
p_{n}^{-}(\psi)=v_{n} \psi, \quad v_{n}=-p_{n-1}^{-}\left(\omega_{1}\right), \quad n \geqq 2 .
$$

This formulation will be of great importance for us and since, as far as we know, an explicit proof of it was not given in [3] or elsewhere we shall give a (formal) proof now.

For $n \geqq 1$ we expand the differential part of $L^{n}$ in powers of $L$ [1-3],

$$
\left(L^{n}\right)_{+}=L^{n}+\sum_{j=1}^{\infty} \sigma_{J}^{(n)} L^{-j}
$$

in particular, the expansion of $\partial_{x}$ becomes

$$
\partial_{x}=L+\sum_{j=1}^{\infty} \sigma_{j}^{(1)} L^{-j}
$$

Applying both sides of (2.9) to $\psi$ and using the linear problem (2.2) gives an expansion

$$
\psi t_{n}=\left(\lambda^{n}+\sum_{j=1}^{\infty} \sigma_{j}^{(n)} \lambda^{-j}\right) \psi
$$

Differentiation of the expression (2.6) leads to

$$
\psi_{t_{n}}=\left(\lambda^{n}-\frac{\tau_{t_{n}}(t)}{\tau(t)}+\frac{\tau_{t_{n}}(t-\varepsilon(\lambda))}{\tau(t-\varepsilon(\lambda))}\right) \psi,
$$

which gives upon Taylor expansion

$$
\psi t_{n}=\left(\lambda^{n}+\sum_{j=1}^{\infty} p_{J}^{-}\left(\frac{\tau_{t_{n}}}{\tau}\right) \lambda^{-j}\right) \psi
$$

If we compare the expansions (2.11) and (2.13) of $\psi_{t_{n}} / \psi$ we obtain

$$
\sigma_{j}^{(n)}=\left(p_{j}^{-}(\log \tau)\right)_{t_{n}} .
$$


As a simple consequence of (2.14) we derive

$$
\left(\sigma_{j}^{(n)}\right)_{x}=\left(\sigma_{j}^{(1)}\right)_{t_{n}},
$$

and using $\omega_{1}=-\tau_{x} / \tau$,

$$
\sigma_{j}^{(1)}=-p_{j}^{-}\left(\omega_{1}\right)=v_{j+1}
$$

From (2.10) it follows

$$
\begin{aligned}
\left(\partial_{x} L^{n}\right)_{+} & =\left(L^{n+1}+\sum_{j=1}^{\infty} \sigma_{j}^{(1)} L^{n-j}\right)_{+} \\
& =\left(L^{n+1}\right)_{+}+\sum_{j=1}^{\infty} \sigma_{j}^{(1)}\left(L^{n-j}\right)_{+}
\end{aligned}
$$

On the other hand, (2.9) gives

$$
\begin{aligned}
\left(\partial_{x} L^{n}\right)_{+} & =\left(\partial_{x}\left(\left(L^{n}\right)_{+}-\sum_{j=1}^{\infty} \sigma_{j}^{(n)} L^{-j}\right)\right)_{+} \\
& =\partial_{x}\left(L^{n}\right)_{+}-\sigma_{1}^{(n)}
\end{aligned}
$$

If we use (2.17), (2.18) and (2.16) we are led to

$$
\partial_{x}\left(L^{n}\right)_{+}=\left(L^{n+1}\right)_{+}+\sum_{j=1}^{\infty} v_{j+1}\left(L^{n-j}\right)_{+}+\sigma_{1}^{(n)} .
$$

Applying (2.19) to $\psi$ gives

$$
\begin{aligned}
\psi_{x t_{n}} & =\psi_{t_{n+1}}+\sum_{j=1}^{\infty} v_{j+1} \psi_{t_{n-\jmath}}+\sigma_{1}^{(n)} \psi \\
& =\psi_{t_{n+1}}+\sum_{j=2}^{\infty} v_{j} \psi_{t_{n+1-\jmath}}+\sigma_{1}^{(n)} \psi
\end{aligned}
$$

In what follows we shall need a property of the polynomials $p_{n}(t)$ which is a consequence of the well-known relation $p_{i}(t)_{t_{m}}=p_{i-m}$, namely

$$
n p_{n}(t)=\sum_{l=1}^{n} l t_{l} p_{n-l}(t)
$$

Equation (2.21) gives

$$
(n+1) p_{n+1}^{-}=-\sum_{j=0}^{n} \partial_{t_{n+1-j}} p_{j}^{-}
$$

Now we shall proceed by mathematical induction. First, we have to check (2.8) for $n=2$. This can be done by using (2.20) for $n=1$. Assume that

$$
p_{J}^{-}(\psi)=v_{j} \psi
$$


holds for $j=3, \ldots, n$. Then we obtain for $j=n+1$ by using (2.22), (2.23) and (2.20),

$$
\begin{aligned}
(n+1) p_{n+1}^{-}(\psi) & =-\sum_{j=0}^{n} \partial_{t_{n+1-j}} p_{j}^{-}(\psi) \\
& =\left(-\partial_{t_{n+1}}+\partial_{t_{n}} \partial_{x}\right) \psi-\sum_{j=2}^{n} \partial_{t_{n+1-j}}\left(v_{j} \psi\right) \\
& =\psi_{x t_{n}}-\psi_{t_{n+1}}-\sum_{j=2}^{n} v_{j} \psi_{t_{n+1-j}}-\sum_{j=2}^{n} v_{j, t_{n+1-j}} \psi \\
& =\left(v_{n+1}+\sigma_{1}^{(n)}-\sum_{j=2}^{n} v_{j, t_{n+1-j}}\right) \psi
\end{aligned}
$$

Through (2.14-2.16) and (2.22), Eq. (2.24) becomes

$$
\begin{aligned}
(n+1) p_{n+1}^{-}(\psi) & \left.\left.=v_{n+1} \psi+\left(p_{1}^{-}(\log \tau)\right)_{t_{n}}-\sum_{j=2}^{n} p_{j-1}^{-}(\log \tau)\right)_{x t_{n+1-}}\right) \psi \\
& =v_{n+1} \psi+\left(-\partial_{t_{n}}-\sum_{j=1}^{n-1} p_{j}^{-}\right)(\log \tau)_{x} \psi \\
& =v_{n+1} \psi+\left(n p_{n}^{-}(\log \tau)\right)_{x} \psi \\
& =(n+1) v_{n+1} \psi
\end{aligned}
$$

which completes the proof.

\section{Consequences of the Linear Problems}

In this section we shall derive two consequences from the linear equations (2.8) which will be very helpful in what follows.

Lemma 1. For $n \geqq 2$ and $j \geqq 1$, it holds:

$$
-p_{n}^{+}\left(\omega_{j}\right)+p_{n-1}^{+}\left(\omega_{j, x}\right)+p_{n-1}^{+}\left(\omega_{j+1}\right)=\sum_{l=0}^{n-1} p_{n-1-l}^{+}\left(\omega_{1}\right) p_{l}^{+}\left(\omega_{j}\right)
$$

Proof. From (2.22) one obtains through mathematical induction

$$
p_{j}^{-}\left(e^{\xi(t, \lambda)}\right)=\left\{\begin{array}{rl}
e^{\xi(t, \lambda)}, & j=0 \\
-\lambda e^{\xi(t, \lambda)}, & j=1 \\
0, & j \geqq 2
\end{array} .\right.
$$

Now we insert the wave function $\psi=\widehat{W} e^{\xi(t, \lambda)}$ into (2.8) and use (3.2) yielding

$$
p_{n}^{-}(\widehat{W})-\lambda p_{n-1}^{-}(\widehat{W})=-p_{n-1}^{-}\left(\omega_{1}\right)(\widehat{W}) .
$$

Comparing coefficients of powers of $\lambda^{-j}$ in (3.3) gives

$$
p_{n}^{-}\left(\omega_{j}\right)-p_{n-1}^{-}\left(\omega_{j+1}\right)=-p_{n-1}^{-}\left(\omega_{1}\right) \omega_{j} .
$$


Multiplying (3.4) by $\lambda^{-n}$ and summing up afterwards shows that

$$
\sum_{n=2}^{\infty} p_{n}^{-}\left(\omega_{j}\right) \lambda^{-n}-\sum_{n=2}^{\infty} p_{n-1}^{-}\left(\omega_{j+1}\right) \lambda^{-n}=-\sum_{n=2}^{\infty} p_{n-1}^{-}\left(\omega_{1}\right) \omega_{j} \lambda^{-n}
$$

Upon Taylor expansion (2.7), Eq. (3.5) can be written as

$$
\begin{aligned}
& \left(e^{\xi(-\tilde{\partial}, \hat{\imath})}-1+\lambda^{-1} \partial_{x}\right)\left(\omega_{j}\right)-\lambda^{-1}\left(e^{\xi(-\tilde{\partial}, \lambda)}-1\right)\left(\omega_{j+1}\right) \\
& =-\lambda^{-1}\left(e^{\xi(-\tilde{\partial}, \hat{\lambda})}-1\right)\left(\omega_{1}\right) \omega_{j} .
\end{aligned}
$$

The formula (2.7) for Taylor expansion yields

$$
e^{\xi(-\tilde{\partial}, \hat{\lambda})}(f(t) g(t))=e^{\xi(-\tilde{\partial}, \hat{\lambda})}(f(t)) e^{\xi(-\tilde{\partial}, \lambda)}(g(t)) .
$$

(An analogous rule holds if we replace $-\varepsilon(\lambda)$ by $\varepsilon(\lambda)$ and $e^{\xi(-\tilde{\partial}, \lambda)}$ by $e^{\xi(\tilde{\partial}, \lambda)}$ ). Application of the Taylor operator $e^{\xi(\tilde{\partial}, \lambda)}$ to (3.6) and using (3.7) yields

$$
\begin{aligned}
& \left(1-e^{\xi(\tilde{\partial}, \lambda)}+\lambda^{-1} e^{\xi(\tilde{\partial}, \lambda)} \partial_{x}\right)\left(\omega_{j}\right)-\lambda^{-1}\left(1-e^{\xi(\tilde{\partial}, \lambda)}\right)\left(\omega_{j+1}\right) \\
& =-\lambda^{-1}\left(1-e^{\xi(-\tilde{\partial}, \lambda)}\right)\left(\omega_{1}\right) e^{\xi(\tilde{\partial}, \lambda)}\left(\omega_{j}\right)
\end{aligned}
$$

Working out the Taylor series in (3.8), leads to

$$
\begin{aligned}
& -\sum_{n=1}^{\infty} p_{n}^{+}\left(\omega_{j}\right) \lambda^{-n}+\sum_{n=0}^{\infty} p_{n}^{+}\left(\omega_{J, x}\right) \lambda^{-(n+1)}+\sum_{n=1}^{\infty} p_{n}^{+}\left(\omega_{j+1}\right) \lambda^{-(n+1)} \\
& =-\lambda^{-1}-\sum_{n=1}^{\infty} p_{n}^{+}\left(\omega_{1}\right) \lambda^{-n} \sum_{n=0}^{\infty} p_{n}^{+}\left(\omega_{j}\right) \lambda^{-n} \\
& =-\lambda^{-2}-\sum_{n=0}^{\infty}\left(\sum_{l=0}^{n} p_{n+1-l}^{+}\left(\omega_{1}\right) p_{l}^{+}\left(\omega_{j}\right)\right) \lambda^{-n}
\end{aligned}
$$

which gives (3.1).

Lemma 2. For $n \geqq 2$ and $j \geqq 1$, it holds

$$
\omega_{j+1, t_{n}}=-p_{n+1}^{+}\left(\omega_{j}\right)+\omega_{j, t_{n+1}}+\omega_{1, t_{n}} \omega_{j}+\sum_{i=1}^{n-1} \omega_{1, t_{n-i}} p_{l}^{+}\left(\omega_{j}\right) .
$$

Proof. From the property (2.21) of the Schur polynomials we have similar to (2.22)

$$
n p_{n}^{+}=\sum_{l=1}^{n} \partial_{t_{l}} p_{n-l}^{+}
$$

yielding

$$
\omega_{j+1, t_{n}}=n p_{n}^{+}\left(\omega_{j+1}\right)-\sum_{l=1}^{n-1} \partial_{t_{l}} p_{n-l}^{+}\left(\omega_{j+1}\right)
$$

Furthermore, we shall use Lemma 1, Eq. (3.1), for indices $h=2,3, \ldots, n+1$,

$$
p_{h-1}^{+}\left(\omega_{j+1}\right)=\sum_{l=0}^{h-2} p_{h-1-l}^{+}\left(\omega_{1}\right) p_{l}^{+}\left(\omega_{j}\right)+p_{h}^{+}\left(\omega_{j}\right)-p_{h-1}^{+}\left(\omega_{j, x}\right) \text {. }
$$


Plugging in (3.13) into (3.12) gives

$$
\begin{aligned}
\omega_{j+1, t_{n}}= & \left(\sum_{l=0}^{n-1} p_{n-i}^{+}\left(\omega_{1}\right) p_{i}^{+}\left(\omega_{j}\right)+p_{n+1}^{+}\left(\omega_{j}\right)-p_{n}^{+}\left(\omega_{j, x}\right)\right) \\
& -\sum_{l=1}^{n-1} \partial_{t_{l}}\left(\sum_{i=0}^{n-l-1} p_{n-l-l}^{+}\left(\omega_{1}\right) p_{i}^{+}\left(\omega_{j}\right)+p_{n-l+1}^{+}\left(\omega_{j}\right)-p_{n-l}^{+}\left(\omega_{j, x}\right)\right) \\
= & n p_{n+1}^{+}\left(\omega_{j}\right)-n p_{n}^{+}\left(\omega_{j, x}\right)+n \sum_{l=0}^{n-1} p_{n-i}^{+}\left(\omega_{1}\right) p_{l}^{+}\left(\omega_{j}\right) \\
& -\sum_{l=1}^{n-1} \sum_{i=0}^{n-l-1}\left(\partial_{t_{l}} p_{n-l-i}^{+}\left(\omega_{1}\right)\right) p_{i}^{+}\left(\omega_{j}\right) \\
& -\sum_{l=1}^{n-1} \sum_{i=0}^{n-l-1} p_{n-l-i}^{+}\left(\omega_{1}\right) \partial_{t_{l}} p_{i}^{+}\left(\omega_{j}\right) \\
& -\sum_{l=1}^{n-1} \partial_{t_{l}} p_{n-l+1}^{+}\left(\omega_{j}\right)+\sum_{l=1}^{n-1} \partial_{t_{l}} p_{n-l}^{+}\left(\omega_{j, x}\right) .
\end{aligned}
$$

If we change the order of summations and use (3.11), Eq. (3.14) reads

$$
\begin{aligned}
\omega_{j+1, t_{n}=} & n p_{n+1}^{+}\left(\omega_{j}\right)-n p_{1}^{+} p_{n}^{+}\left(\omega_{j}\right)+n \sum_{i=0}^{n-1} p_{n-l}^{+}\left(\omega_{1}\right) p_{i}^{+}\left(\omega_{j}\right) \\
& -\sum_{i=0}^{n-2}\left(\sum_{l=1}^{n-i-1} \partial_{t_{l}} p_{n-l-i}^{+}\left(\omega_{1}\right)\right) p_{i}^{+}\left(\omega_{j}\right)-\sum_{m=1}^{n-1} \sum_{l=1}^{m} p_{n-m}^{+}\left(\omega_{1}\right) \partial_{t_{l}} p_{m-l}^{+}\left(\omega_{j}\right) \\
& -(n+1) p_{n+1}^{+}\left(\omega_{j}\right)+\partial_{t_{n}} p_{1}^{+}\left(\omega_{j}\right)+\partial_{t_{n+1}}\left(\omega_{j}\right)+\left(n p_{n}^{+}-\partial_{t_{n}}\right) p_{1}^{+}\left(\omega_{j}\right) \\
= & -p_{n+1}^{+}\left(\omega_{j}\right)+\partial_{t_{n+1}}\left(\omega_{j}\right)+\partial_{t_{n}}\left(\omega_{1}\right) \omega_{j} \\
& +\sum_{l=1}^{n-2}\left(n p_{n-i}^{+}\left(\omega_{1}\right)-\sum_{l=1}^{n-l-1} \partial_{t_{l}} p_{n-l-i}^{+}\left(\omega_{1}\right)-i p_{n-l}^{+}\left(\omega_{1}\right)\right) p_{i}^{+}\left(\omega_{j}\right) \\
& +p_{1}^{+}\left(\omega_{1}\right) p_{n-1}^{+}\left(\omega_{j}\right) \\
= & -p_{n+1}^{+}\left(\omega_{j}\right)+\partial_{t_{n+1}}\left(\omega_{j}\right)+\partial_{t_{n}}\left(\omega_{1}\right) \omega_{j} \\
& +\sum_{i=1}^{n-2}\left((n-i) p_{n-i}^{+}\left(\omega_{1}\right)-\sum_{l=1}^{n-i-1} \partial_{t_{l}} p_{n-l-i}^{+}\left(\omega_{1}\right)\right) p_{i}^{+}\left(\omega_{j}\right)+p_{1}^{+}\left(\omega_{1}\right) p_{n-1}^{+}\left(\omega_{j}\right) \\
= & -p_{n+1}^{+}\left(\omega_{J}\right)+\omega_{j, t_{n+1}}+\omega_{1, t_{n}} \omega_{j}+\sum_{l=1}^{n-1} \omega_{1, t_{n-l}} p_{i}^{+}\left(\omega_{j}\right)
\end{aligned}
$$

\section{Sato's Equation and Constraints}

It is more convenient to impose the constraint (1.18) on Sato's equations

$$
W_{t_{n}}=-\left(L^{n}\right)_{-} W
$$

than on the Lax-equation (1.14), 
Proposition 1. Under the constraint (1.18) we have for $j \geqq 0$,

$$
\omega_{j+1, t_{k}}+\omega_{j, t_{k} x}+q r \omega_{j}=\frac{q_{x}}{q} \omega_{j, t_{k}} .
$$

Proof. From (4.1) and (1.18) it follows

$$
W_{t_{k}}=-q \partial^{-1} r W
$$

Now we introduce an intermediate operator

$$
N=\partial^{-1} r W=n_{1} \partial^{-1}+n_{2} \partial^{-2}+n_{3} \partial^{-3}+\cdots, \quad n_{1}=r .
$$

The definition of $N$ gives

$$
q N \partial+q N_{x}=q r W .
$$

From Sato's equation (4.1) we obtain

$$
W_{t_{k}}=-q N
$$

From Eq. (4.5) and (4.6) one obtains for $j \geqq 0$,

$$
\begin{gathered}
q n_{j+1}+q n_{j, x}=q r \omega_{j}, \\
\omega_{j, t_{k}}=-q n_{j} .
\end{gathered}
$$

Finally, elimination of the intermediate operator $N$ gives the claim. From Eq. (4.8) we get

$$
\begin{aligned}
q n_{j, x} & =\left(q n_{j}\right)_{x}-q_{x} n_{j} \\
& =\left(q n_{j}\right)_{x}-\frac{q x}{q} q n_{j} \\
& =-\omega_{j, t_{k} x}+\frac{q_{x}}{q} \omega_{j, t_{x}},
\end{aligned}
$$

and

$$
q n_{j+1}=-\omega_{j+1, t_{k}} .
$$

Introducing (4.9) and (4.10) into (4.7) gives (4.2).

\section{The 1-Constraint}

Let us define a sequence $a_{n}, n \geqq 1$ through:

$$
a_{1}=1, a_{2}=q_{x} / q \text { and for } n \geqq 3 \text {, }
$$

$$
a_{n}=a_{n-1, x}+\frac{q_{x}}{q} a_{n-1}-\sum_{l=1}^{n-2} p_{n-1-l}^{+}\left(\omega_{1}\right) a_{l} .
$$

As an example we note

$$
a_{3}=\left(\frac{q_{x}}{q}\right)_{x}+\left(\frac{q_{x}}{q}\right)^{2}+q r .
$$


Proposition 2. In the case of 1-constraint, $L_{-}=q \partial^{-1} r$, we have for any $j \geqq 1$ and $n \geqq 1$,

$$
p_{n}^{+}\left(\omega_{j}\right)=a_{n} \omega_{j, x}
$$

Proof. We shall proceed by mathematical induction. It is clear that

$$
p_{1}^{+}\left(\omega_{J}\right)=a_{1} \omega_{j, x}
$$

Now suppose

$$
p_{l}^{+}\left(\omega_{J}\right)=a_{l} \omega_{j, x}, \quad l=2, \ldots, n-1 .
$$

Using (3.1) and (5.3) we obtain

$$
-p_{n}^{+}\left(\omega_{j}\right)+\left(a_{n-1} \omega_{j, x}\right)_{x}+a_{n-1} \omega_{j+1, x}=p_{n-1}^{+}\left(\omega_{1}\right) \omega_{j}+\sum_{l=1}^{n-2} p_{n-1-l}^{+}\left(\omega_{1}\right) a_{l} \omega_{j, x} .
$$

Note that Eq. (4.3) gives $\omega_{1, t_{k}}=-q r$, in particular, $\omega_{1, x}=-q r$ in the case of 1-constraint. Using the last equation together with (5.3), we can write

$$
p_{n-1}^{+}\left(\omega_{1}\right)=a_{n-1} \omega_{1, x}=-a_{n-1} q r \text {. }
$$

Taking (5.5) into account as well as (4.2) for $k=1$, Eq. (5.4) becomes

$$
\begin{aligned}
p_{n}^{+}\left(\omega_{j}\right) & =\left(a_{n-1, x}-\sum_{l=1}^{n-2} p_{n-1-l}^{+}\left(\omega_{1}\right) a_{l}\right) \omega_{j, x}+a_{n-1}\left(\omega_{j+1, x}+\omega_{j, x x}+q r \omega_{j}\right) \\
& =\left(a_{n-1, x}+\frac{q_{x}}{q} a_{n-1}-\sum_{l=1}^{n-2} p_{n-1-l}^{+}\left(\omega_{1}\right) a_{l}\right) \omega_{j, x} \\
& =a_{n} \omega_{j, x},
\end{aligned}
$$

i.e. (5.2).

Let us write Eq. (5.2) as

$$
p_{1}^{+}\left(\omega_{J}\right) a_{n}+p_{n}^{+}\left(\omega_{j}\right)(-1)=0
$$

and consider (5.6) for two different indices $i$ and $j$ as a linear equation with a nontrivial solution, then we obtain the following:

Corollary. For $n \geqq 2$ we have

$$
\left|\begin{array}{ll}
p_{1}^{+}\left(\omega_{i}\right) & p_{1}^{+}\left(\omega_{j}\right) \\
p_{n}^{+}\left(\omega_{l}\right) & p_{n}^{+}\left(\omega_{j}\right)
\end{array}\right|=0
$$

for arbitrary $i, j \geqq 1$.

From Taylor expansion (2.7) we obtain the rule

$$
p_{n}^{+}(f(t) g(t))=\sum_{l=0}^{n} p_{l}^{+}(f(t)) p_{n-l}^{+}(g(t)) .
$$

(An analogous rule holds if we replace $\varepsilon(\lambda)$ by $-\varepsilon(\lambda)$ and $p_{n}^{+}$by $p_{n}^{-}$.) With the rule (5.8) and (1.25) we calculate

$$
p_{n}^{+}\left(\omega_{m}\right)=p_{n}^{+}\left(\frac{1}{\tau} p_{m}^{-}(\tau)\right)=\sum_{l=0}^{n} p_{n-l}^{+}\left(\frac{1}{\tau}\right) p_{l}^{+}\left(p_{m}^{-}(\tau)\right)
$$


This then shows that the $2 \times 2$-determinants (5.7) represent multilinear forms in $\tau$. They describe the set of $\tau$-functions of the AKNS hierarchy. Considering the $2 \times 2$ determinants (5.7) with the lowest indices $i=1, j=2$ we obtain for each index $n$ a flow involving the time variables $t_{2}, t_{3}, \ldots, t_{n}$.

\section{Trilinear Forms for the 1-Constraint}

The following proposition shows that the multilinear forms (5.7) can be changed to trilinear forms.

Proposition 3. The set of Eq. (5.7), where $n \geqq 2$ and $i, j \geqq 1$, implies the following set of equations:

$$
\left|\begin{array}{ccc}
p_{j 1}^{+} p_{i 1}^{-}(\tau) & p_{j 1}^{+} p_{i 2}^{-}(\tau) & p_{j 1}^{+} p_{i 3}^{-}(\tau) \\
p_{j 2}^{+} p_{i 1}^{-}(\tau) & p_{j 2}^{+} p_{i 2}^{-}(\tau) & p_{j 2}^{+} p_{i 3}^{-}(\tau) \\
p_{j 3}^{+} p_{i 1}^{-}(\tau) & p_{j 3}^{+} p_{i 2}^{-}(\tau) & p_{j 3}^{+} p_{i 3}^{-}(\tau)
\end{array}\right|=0
$$

where $i_{\mu} \geqq 0$ and $j_{\mu} \geqq 0, \mu=1,2,3$, are arbitrary indices.

Proof. Using the rule (5.8) we prepare

$$
p_{n}^{+}\left(\frac{1}{\tau} \tau\right)=\sum_{l=0}^{n} p_{n-l}^{+}\left(\frac{1}{\tau}\right) p_{l}^{+}(\tau)=0
$$

The vanishing of the determinant (5.7) means that there are functions $b_{n i j}=b_{n i j}(t)$ with

$$
\left(\begin{array}{c}
p_{n}^{+}\left(\omega_{j}\right) \\
p_{n}^{+}\left(\omega_{j}\right)
\end{array}\right)=b_{n i j}\left(\begin{array}{c}
p_{1}^{+}\left(\omega_{i}\right) \\
p_{1}^{+}\left(\omega_{j}\right)
\end{array}\right)
$$

We extend this through

$$
\left(\begin{array}{c}
0 \\
p_{n}^{+}\left(\omega_{i}\right) \\
p_{n}^{+}\left(\omega_{j}\right)
\end{array}\right)=b_{n i j}\left(\begin{array}{c}
0 \\
p_{1}^{+}\left(\omega_{l}\right) \\
p_{1}^{+}\left(\omega_{j}\right)
\end{array}\right)
$$

and obtain

$$
\left(\begin{array}{c}
p_{n}^{+}\left(\frac{1}{\tau} \tau\right) \\
p_{n}^{+}\left(\frac{1}{\tau} p_{i}^{-}(\tau)\right) \\
p_{n}^{+}\left(\frac{1}{\tau} p_{j}^{-}(\tau)\right)
\end{array}\right)=b_{n i j}\left(\begin{array}{c}
p_{1}^{+}\left(\frac{1}{\tau} \tau\right) \\
p_{1}^{+}\left(\frac{1}{\tau} p_{i}^{-}(\tau)\right) \\
p_{1}^{+}\left(\frac{1}{\tau} p_{j}^{-}(\tau)\right)
\end{array}\right)
$$

Now, making use of (5.8) and (6.2) gives

$$
\sum_{l=0}^{n} p_{n-l}^{+}\left(\frac{1}{\tau}\right)\left(\begin{array}{c}
p_{l}^{+}(\tau) \\
p_{l}^{+}\left(p_{l}^{-}(\tau)\right) \\
p_{l}^{+}\left(p_{j}^{-}(\tau)\right)
\end{array}\right)=b_{n i j} \sum_{l=0}^{1} p_{1-l}^{+}\left(\frac{1}{\tau}\right)\left(\begin{array}{c}
p_{l}^{+}(\tau) \\
p_{l}^{+}\left(p_{l}^{-}(\tau)\right) \\
p_{l}^{+}\left(p_{j}^{-}(\tau)\right)
\end{array}\right)
$$


which can be written as

$$
\begin{aligned}
& \left(p_{n}^{+}\left(\frac{1}{\tau}\right)-b_{n i j} p_{1}^{+}\left(\frac{1}{\tau}\right)\right)\left(\begin{array}{c}
p_{0}^{+}(\tau) \\
p_{0}^{+}\left(p_{l}^{-}(\tau)\right) \\
p_{0}^{+}\left(p_{j}^{-}(\tau)\right)
\end{array}\right) \\
& \quad+\left(p_{n-1}^{+}\left(\frac{1}{\tau}\right)-b_{n i j} p_{0}^{+}\left(\frac{1}{\tau}\right)\right)\left(\begin{array}{c}
p_{1}^{+}(\tau) \\
p_{1}^{+}\left(p_{i}^{-}(\tau)\right) \\
p_{1}^{+}\left(p_{j}^{-}(\tau)\right)
\end{array}\right) \\
& +\sum_{l=2}^{n} p_{n-l}^{+}\left(\frac{1}{\tau}\right)\left(\begin{array}{c}
p_{l}^{+}(\tau) \\
p_{l}^{+}\left(p_{l}^{-}(\tau)\right) \\
p_{l}^{+}\left(p_{j}^{-}(\tau)\right)
\end{array}\right)=0 .
\end{aligned}
$$

Considering Eq.(6.6) for arbitrary $n \geqq 2$ shows that we can express any vector $l \geqq 2$

through the vectors

$$
\left(\begin{array}{c}
p_{l}^{+}(\tau) \\
p_{l}^{+}\left(p_{i}^{-}(\tau)\right) \\
p_{l}^{+}\left(p_{j}^{-}(\tau)\right)
\end{array}\right)
$$

$$
\left(\begin{array}{c}
p_{0}^{+}(\tau) \\
p_{0}^{+}\left(p_{l}^{-}(\tau)\right) \\
p_{0}^{+}\left(p_{J}^{-}(\tau)\right)
\end{array}\right), \quad\left(\begin{array}{c}
p_{1}^{+}(\tau) \\
p_{1}^{+}\left(p_{l}^{-}(\tau)\right) \\
p_{1}^{+}\left(p_{J}^{-}(\tau)\right)
\end{array}\right)
$$

So any three of the vectors

$$
\left(\begin{array}{c}
p_{l}^{+}(\tau) \\
p_{l}^{+}\left(p_{l}^{-}(\tau)\right) \\
p_{l}^{+}\left(p_{j}^{-}(\tau)\right)
\end{array}\right)
$$

are linearly dependent. Taking the arbitrariness of the indices $i, j$ into account, this means that

$$
\left|\begin{array}{lll}
p_{j 1}^{+} p_{0}^{-}(\tau) & p_{j 1}^{+} p_{i 2}^{-}(\tau) & p_{j 1}^{+} p_{l 3}^{-}(\tau) \\
p_{j 2}^{+} p_{0}^{-}(\tau) & p_{j 2}^{+} p_{l 2}^{-}(\tau) & p_{j 2}^{+} p_{l 3}^{-}(\tau) \\
p_{j 3}^{+} p_{0}^{-}(\tau) & p_{j 3}^{+} p_{l 2}^{-}(\tau) & p_{j 3}^{+} p_{l 3}^{-}(\tau)
\end{array}\right|=0
$$

And from (6.7) again by using linear algebra we obtain the proposition.

The proof of Proposition 3 shows that the basic $2 \times 2$ matrix

$$
\left|\begin{array}{ll}
p_{1}^{+}\left(\omega_{1}\right) & p_{1}^{+}\left(\omega_{2}\right) \\
p_{n}^{+}\left(\omega_{1}\right) & p_{n}^{+}\left(\omega_{2}\right)
\end{array}\right|=0
$$

implies the well-known trilinear form (1.5) of the Kaup-Broer system. (Here basic means that the flow corresponding to (6.8) involves the lowest possible number of time variables, namely $t_{2}$.)

\section{The k-Constraint, Basic Flow}

Let us first consider the case of 1-constraint, $k=1$, and fix the index $n=2$. Lemma 1, Equation (3.1), then may be used for eliminating $\omega_{j+1, x}$ in Proposition 1, Equation (4.2). This gives us the $2 \times 2$-determinants (5.7), (where $n=2$ ), and finally, we end up with the basic determinants (6.8). In the case of k-constraints, we shall now 
try to find determinants which similar to $(5.7)$ (with $n=2$ ) give rise to basic flows, where basic now means that the flow involves the time variables $t_{2}, \ldots, t_{k+1}$.

Proposition 4. Under the condition of $k$-constraint (1.18), $k \geqq 2$, for $j \geqq 1$ the following holds:

$$
\sum_{l=1}^{k-1} p_{i}^{+}\left(\omega_{j}\right) \omega_{1, t_{k-i}}-\omega_{j, t_{k}} \frac{q_{x}}{q}+T_{k+1}^{(k)}\left(\omega_{j}\right)=0
$$

where

$$
T_{k+1}^{(k)}=-p_{k+1}^{+}+\partial_{t_{k+1}}+\partial_{x} \partial_{t_{k}}
$$

Proof. Use Proposition 1, Eq. (4.2), and Lemma 2, Eq. (3.10) with $n=k$, ie.,

$$
\omega_{j+1, t_{k}}=-p_{k+1}^{+}\left(\omega_{j}\right)+\omega_{j, t_{k+1}}+\omega_{1, t_{k}} \omega_{J}+\sum_{i=1}^{k-1} \omega_{1, t_{k-l}} p_{i}^{+}\left(\omega_{j}\right) .
$$

Inserting (7.3) into (4.2) and taking $\omega_{1, t_{k}}=-q r$ into account gives the claim.

Note that in the case $k=1$, for $n=2$, Eq. (5.2) becomes

$$
-\omega_{j, x} \frac{q_{x}}{q}+p_{2}^{+}\left(\omega_{j}\right)=0
$$

and that

$$
p_{2}^{+}=-p_{2}^{+}+\partial_{t_{2}}+\partial_{x} \partial_{t_{1}}=T_{2}^{(1)}\left(\omega_{j}\right)
$$

which is consistent with (7.2).

As an example we now consider $k=2$, where (7.1) reads

$$
p_{1}^{+}\left(\omega_{j}\right) \omega_{1, x}-\omega_{j, t_{x}} \frac{q_{x}}{q}+T_{3}^{(2)} \omega_{j}=0
$$

and the linear operator (7.2) becomes

$$
T_{3}^{(2)}=-p_{3}^{+}+\partial_{t_{3}}+\partial_{x} \partial_{t_{2}} .
$$

From Proposition 3 we obtain the following

Corollary. Under the condition of $k$-constraint (1.18), $k \geqq 2$, for an arbitrary set of indices $j_{l}, j_{2}, \ldots, j_{k+1}$ the following holds:

$$
\left|\begin{array}{cccc}
p_{1}^{+}\left(\omega_{j_{1}}\right) & p_{1}^{+}\left(\omega_{j_{2}}\right) & \cdots & p_{1}^{+}\left(\omega_{j_{k+1}}\right) \\
p_{2}^{+}\left(\omega_{j_{1}}\right) & p_{2}^{+}\left(\omega_{j_{2}}\right) & \cdots & p_{2}^{+}\left(\omega_{j_{k+1}}\right) \\
\vdots & \vdots & \vdots & \vdots \\
p_{k-1}^{+}\left(\omega_{j 1}\right) & p_{k-1}^{+}\left(\omega_{j_{2}}\right) & \cdots & p_{k-1}^{+}\left(\omega_{j_{k+1}}\right) \\
\omega_{j_{1}, t_{k}} & \omega_{j_{2}, t_{k}} & \cdots & \omega_{j_{k+1}, t_{k}} \\
T_{k+1}^{(k)}\left(\omega_{j_{1}}\right) & T_{k+1}^{(k)}\left(\omega_{j_{2}}\right) & \cdots & T_{k+1}^{(k)}\left(\omega_{j_{k+1}}\right)
\end{array}\right|=0
$$


Proof. Consider the Eq. (7.1),

$$
\sum_{l=1}^{k-1} p_{i}^{+}\left(\omega_{j_{p}}\right) \omega_{1, t_{k-l}}-\omega_{j_{\rho}, t_{k}} \frac{q_{x}}{q}+T_{k+1}^{(k)} \omega_{j_{p}}=0,
$$

with $\rho=1, \ldots, k+1$ as $(k+1) \times(k+1)$ linear system with the nontrivial solution vector

$$
\left(\begin{array}{c}
\omega_{1, t_{k-1}} \\
\omega_{1, t_{k-2}} \\
\vdots \\
\omega_{1, x} \\
-\frac{q_{x}}{q} \\
1
\end{array}\right) .
$$

As an example we again consider the case $k=2$, where the determinant (7.8) becomes

$$
\left|\begin{array}{ccc}
p_{1}^{+}\left(\omega_{j_{1}}\right) & p_{1}^{+}\left(\omega_{j_{2}}\right) & p_{1}^{+}\left(\omega_{j_{3}}\right) \\
\omega_{j_{1}, t_{2}} & \omega_{j_{2}, t_{2}} & \omega_{j_{3}, t_{2}} \\
T_{3}^{(2)}\left(\omega_{j_{1}}\right) & T_{3}^{(2)}\left(\omega_{j_{2}}\right) & T_{3}^{(2)}\left(\omega_{j_{3}}\right)
\end{array}\right|=0 .
$$

In accordance with (7.5) the determinant (7.8) becomes for the 1-constraint $k=1$ :

$$
\left|\begin{array}{cc}
p_{1}^{+}\left(\omega_{j_{1}}\right) & p_{1}^{+}\left(\omega_{j_{2}}\right) \\
T_{2}^{(1)}\left(\omega_{j_{1}}\right) & T_{2}^{(1)}\left(\omega_{j_{2}}\right)
\end{array}\right|=0
$$

Choosing the indices $j_{1}, j_{2}, \ldots, j_{k+1}$ as basic ones, i.e. $1,2, \ldots, k+1$, we obtain flows for k-constraints involving time variables $t_{2}, t_{3}, \ldots, t_{k+1}$.

\section{The k-Constraint, Higher Flows}

We now aim at obtaining the next higher flows for k-constraints, $k \geqq 2$, i.e., flows in forms of determinants like (7.8) which involve time variables $t_{2}, t_{3}, \ldots, t_{k+2}, t_{k+3}$ after choosing basic indices.

Proposition 5. Under the condition of $k$-constraint (1.18), $k \geqq 2$, for $j \geqq 1$ the following holds:

$$
\begin{aligned}
& \sum_{i=1}^{k-1}\left(\omega_{1, x t_{k-l}}+p_{k+1-i}^{+}\left(\omega_{1}\right)\right) p_{i}^{+}\left(\omega_{j}\right)+\sum_{i=1}^{k-1} \omega_{1, t_{k-i}} \sum_{l=1}^{l-1} p_{l-l}^{+}\left(\omega_{1}\right) p_{l}^{+}\left(\omega_{j}\right) \\
& \quad+\sum_{i=1}^{k-2} \omega_{1, t_{k-l}} p_{i+1}^{+}\left(\omega_{J}\right)+\sum_{i=1}^{k-1} \omega_{1, t_{k+1-i}} p_{i}^{+}\left(\omega_{j}\right) \\
& +\omega_{1, t_{k}} \omega_{j, x}+\omega_{1, x} p_{k}^{+}\left(\omega_{J}\right)-\left(\frac{q_{x}}{q}\right)^{2} \omega_{j, t_{k}}+\frac{q_{x}}{q} \omega_{j, x t_{k}}+T_{k+2}^{(k)}\left(\omega_{j}\right)=0,
\end{aligned}
$$


where

$$
T_{k+2}^{(k)}=-2 p_{k+2}^{+}+\partial_{t_{k+2}}+\partial_{x} \partial_{t_{k+1}}
$$

Proof. We shall use the shorthand notation

$$
a=-\frac{q x}{q}, \quad b_{i}=\omega_{1, t_{k-\imath}}
$$

and the convention

$$
\sum_{l=i}^{j} \cdots=0
$$

if $j \leqq i$.

For $n=k+2$, Lemma 1. Eq. (3.1), reads

$$
-p_{k+2}^{+}\left(\omega_{j}\right)+p_{k+1}^{+}\left(\omega_{j, x}\right)+p_{k+1}^{+}\left(\omega_{j+1}\right)-\sum_{l=0}^{k} p_{k+1-i}^{+}\left(\omega_{1}\right) p_{i}^{+}\left(\omega_{j}\right)=0 .
$$

Next, use Proposition 4, Eq. (7.2), to remove $p_{k+1}^{+}\left(\omega_{j+1}\right)$, obtaining

$$
\begin{gathered}
-p_{k+2}^{+}\left(\omega_{j}\right)+p_{k+1}^{+}\left(\omega_{j, x}\right)+a \omega_{j+1, t_{x}}+\sum_{i=1}^{k-1} b_{i} p_{i}^{+}\left(\omega_{j+1}\right) \\
+\omega_{j+1, x t_{k}}+\omega_{j+1, t_{k+1}}-\sum_{i=0}^{k} p_{k+1-i}^{+}\left(\omega_{1}\right) p_{l}^{+}\left(\omega_{j}\right)=0 .
\end{gathered}
$$

Through application of Lemma 1, Eq. (3.1), Lemma 2, Eq. (3.10), and Proposition 1, Eq. (4.2), Eq. (8.5) becomes

$$
\begin{aligned}
& -p_{k+2}^{+}\left(\omega_{j}\right)+p_{k+1}^{+}\left(\omega_{j, x}\right)+a\left(-a \omega_{j, t_{k}}-\omega_{j, t_{k} x}+\omega_{1, t_{k}} \omega_{j}\right) \\
& +\sum_{l=1}^{k-1} b_{i}\left(\sum_{l=0}^{l-1} p_{i-l}^{+}\left(\omega_{1}\right) p_{l}^{+}\left(\omega_{j}\right)\right)+\sum_{i=0}^{k-1} b_{i} p_{i+1}^{+}\left(\omega_{j}\right)-\sum_{i=0}^{k-1} b_{i} p_{i}^{+}\left(\omega_{j, x}\right) \\
& \quad-p_{k+2}^{+}\left(\omega_{j}\right)+\omega_{j, t_{k+2}}+\sum_{l=0}^{k} \omega_{1}, t_{k+1-i} p_{i}^{+}\left(\omega_{j}\right) \\
& \quad+\left(-a \omega_{j, t_{k}}-\omega_{j, t_{k} x}+\omega_{1}, t_{k} \omega_{j}\right)_{x}-\sum_{i=0}^{k} p_{k+1-i}^{+}\left(\omega_{1}\right) p_{i}^{+}\left(\omega_{j}\right)=0 .
\end{aligned}
$$

By using the convention made above (8.6) gives

$$
\begin{aligned}
& -2 p_{k+2}^{+}\left(\omega_{j}\right)+p_{1}^{+} p_{k+1}^{+}\left(\omega_{j}\right)-a^{2} \omega_{j, t_{k}}-a \omega_{j t_{k} x} \\
& \quad+\sum_{i=1}^{k-1} b_{l}\left(\sum_{l=1}^{i-1} p_{i-l}^{+}\left(\omega_{1}\right) p_{l}^{+}\left(\omega_{j}\right)\right)+\sum_{i=1}^{k-1} b_{i} p_{l+1}^{+}\left(\omega_{j}\right) \\
& \quad-\sum_{i=1}^{k-1} b_{i} p_{1}^{+} p_{i}^{+}\left(\omega_{j}\right)+\omega_{j, t_{k+2}} \sum_{i=1}^{k-1} \omega_{1, t_{k+1-l}} p_{i}^{+}\left(\omega_{j}\right) \\
& \quad-a_{x} \omega_{j, t_{k}}-a \omega_{j, t_{k} x}+\omega_{1}, t_{k} \omega_{j, x}-\omega_{j, t_{k} x x}-\sum_{i=1}^{k} p_{k+1-i}^{+}\left(\omega_{1}\right) p_{i}^{+}\left(\omega_{j}\right)=0 .
\end{aligned}
$$


By using Proposition 4, Eq. (7.1), again Eq. (8.7) becomes

$$
\begin{aligned}
& -2 p_{k+2}^{+}\left(\omega_{j}\right)+a_{x} \omega_{j, t_{k}}+\sum_{i=1}^{k-1} b_{i, x} p_{i}^{+}\left(\omega_{J}\right)+a \omega_{j, t_{k} x}+\sum_{i=1}^{k-1} b_{i} p_{1}^{+} p_{i}^{+}\left(\omega_{j}\right) \\
& +\partial_{t_{x}}\left(\omega_{j, x x}\right)+\omega_{j, t_{k+1} x}-a^{2} \omega_{j, t_{k}}-a \omega_{j t_{k} x}+\sum_{l=1}^{k-1} b_{i} \sum_{l=1}^{i-1} p_{i-l}^{+}\left(\omega_{1}\right) p_{l}^{+}\left(\omega_{j}\right) \\
& +\sum_{i=1}^{k-1} b_{i} p_{i+1}^{+}\left(\omega_{j}\right)-\sum_{l=1}^{k-1} b_{l} p_{1}^{+} p_{l}^{+}\left(\omega_{j}\right)+\partial_{t_{k+2}}\left(\omega_{j}\right)+\sum_{i=1}^{k} \partial_{t_{k+1-l}}\left(\omega_{1}\right) p_{i}^{+}\left(\omega_{j}\right) \\
& -a_{x} \omega_{j, t_{k}}-a \omega_{j, t_{k} x}+\omega_{1, t_{k}} \omega_{j, x}-\omega_{j, t_{k} x x}-\sum_{l=1}^{k} p_{k+1-i}^{+}\left(\omega_{1}\right) p_{i}^{+}\left(\omega_{j}\right)=0
\end{aligned}
$$

which can be arranged to

$$
\begin{aligned}
& \left(-2 p_{k+2}^{+}+\partial_{t_{k+2}}+\partial_{x} \partial_{t_{k+1}}\right) \omega_{j}+\sum_{l=1}^{k-1}\left(b_{l, x}-p_{k+1-l}^{+}\left(\omega_{1}\right)\right) p_{i}^{+}\left(\omega_{j}\right) \\
& \quad+\sum_{i=1}^{k-1} b_{i} \sum_{l=1}^{i-1} p_{i-l}^{+}\left(\omega_{1}\right) p_{l}^{+}\left(\omega_{j}\right)+\sum_{i=1}^{k-2} b_{i} p_{l+1}^{+}\left(\omega_{j}\right)+\sum_{i=1}^{k-1} \omega_{1, t_{k+1-l}} p_{i}^{+}\left(\omega_{j}\right) \\
& \quad+\omega_{1, t_{k}} p_{1}^{+}\left(\omega_{j}\right)+\omega_{1, x} p_{k}^{+}\left(\omega_{j}\right)-a^{2} \omega_{j, t_{k}}-a \omega_{j, t_{k} x}=0,
\end{aligned}
$$

i.e. $(8.1)$.

Taking (8.1) again as a linear equation with non-trivial solutions and using $k+3$ different indices we obtain $(k+3) \times(k+3)$-determinants for $\mathrm{k}$-constrained flows. Those flows involve the time variables $t_{2}, t_{3}, \ldots, t_{k+3}$ if we choose the basic set of indices.

As an example we consider the case $k=2$ where (8.1) becomes

$$
\left(\omega_{1, x x}+2 \omega_{1, t_{2}}+p_{2}^{+}\left(\omega_{1}\right)\right) p_{1}^{+}\left(\omega_{j}\right)+\omega_{1, x} p_{2}^{+}\left(\omega_{j}\right)+\frac{q_{x}}{q} \omega_{j, t_{2} x}+T_{4}^{(2)}\left(\omega_{j}\right)=0,
$$

while (8.2) becomes

$$
T_{4}^{(2)}=-2 p_{4}^{+}+\partial_{t_{4}}+\partial_{x} \partial_{t_{3}} .
$$

As a result from (8.10) we obtain the following $5 \times 5$-determinant flow for 2 constraint

$$
\left|\begin{array}{ccccc}
p_{1}^{+}\left(\omega_{j_{1}}\right) & p_{1}^{+}\left(\omega_{j_{2}}\right) & p_{1}^{+}\left(\omega_{j_{3}}\right) & p_{1}^{+}\left(\omega_{j_{4}}\right) & p_{1}^{+}\left(\omega_{j_{5}}\right) \\
p_{2}^{+}\left(\omega_{j_{1}}\right) & p_{2}^{+}\left(\omega_{j_{2}}\right) & p_{2}^{+}\left(\omega_{j_{3}}\right) & p_{2}^{+}\left(\omega_{j_{4}}\right) & p_{2}^{+}\left(\omega_{j_{5}}\right) \\
\omega_{j_{1}, t_{2}} & \omega_{j_{2}, t_{2}} & \omega_{j_{3}, t_{2}} & \omega_{j_{4}, t_{2}} & \omega_{J_{5}, t_{2}} \\
\omega_{j_{1}, t_{2} x} & \omega_{j_{2}, t_{2} x} & \omega_{j_{3}, t_{2} x} & \omega_{j_{4}, t_{2} x} & \omega_{j_{5}, t_{2} x} \\
T_{4}^{(2)}\left(\omega_{j_{1}}\right) & T_{4}^{(2)}\left(\omega_{j_{2}}\right) & T_{4}^{(2)}\left(\omega_{j_{3}}\right) & T_{4}^{(2)}\left(\omega_{j_{4}}\right) & T_{4}^{(2)}\left(\omega_{j_{5}}\right)
\end{array}\right|=0 .
$$




\section{Concluding Remarks}

This paper was motivated by the discovery of the trilinear form (1.5) for the KaupBroer system. One of our main goals was to derive (1.5) from the point of view of $\mathrm{k}$-constraints (1.18). The idea for this approach to trilinear forms goes back to the fact that the Kaup-Broer system can be transformed to the nonlinear Schrödinger system (basic system of the AKNS hierarchy) which appears as a reduction of the KP hierarchy under the 1-constraint.

It turned out that rather than studying the impact of k-constraints directly on the Lax-equations (1.14) of the KP hierarchy it is more convenient to work with Sato's equations (4.1) which can be solved on the space of $\tau$-functions through (1.25).

Considering Sato's equations under the 1-constraint, we are led first to Eq. (4.3) and in succession to the $2 \times 2$-determinants (1.24). Our claim now was that the trilinear forms (1.23), the simplest of which is (1.5), follow from (1.24) using (1.25). In Sect. 6 we have used several formal assumptions for that derivation, namely: that $p_{n}^{+}(1 / \tau)$ is meaningful and that we can write

$$
p_{n}^{+}\left(\omega_{j}\right)=p_{n}^{+}\left(\frac{1}{\tau} p_{j}^{-}(\tau)\right)=\sum_{l=0}^{n} p_{n-l}^{+}\left(\frac{1}{\tau}\right) p_{l}^{+}\left(p_{j}^{-}(\tau)\right) .
$$

(In the particular case $j=0$ this gives (6.2).)

The question remains open whether or not solutions of (1.23) are lost by these assumptions. (For example, take $\tau=0$ as a solution to (1.23).) However, under the above assumptions we can also obtain (1.24) from (1.23). In order to show this, let us set $j_{1}=0, j_{2}=1, j_{3}=l$ and $i_{1}=0, i_{2}=i, i_{3}=j$ in (1.23) giving

$$
\left|\begin{array}{ccc}
\tau & p_{1}^{+}(\tau) & p_{l}^{+}(\tau) \\
p_{i}^{-}(\tau) & p_{1}^{+}\left(p_{i}^{-}(\tau)\right) & p_{l}^{+}\left(p_{i}^{-}(\tau)\right) \\
p_{j}^{-}(\tau) & p_{1}^{+}\left(p_{j}^{-}(\tau)\right) & p_{l}^{+}\left(p_{j}^{-}(\tau)\right)
\end{array}\right|=0
$$

Writing

$$
\left(\begin{array}{c}
0 \\
p_{n}^{+}\left(\omega_{l}\right) \\
p_{n}^{+}\left(\omega_{j}\right)
\end{array}\right)=\sum_{l=0}^{n} p_{n-l}^{+}\left(\frac{1}{\tau}\right)\left(\begin{array}{c}
p_{l}^{+}(\tau) \\
p_{l}^{+} p_{i}^{-}(\tau) \\
p_{l}^{+} p_{j}^{-}(\tau)
\end{array}\right)
$$

we obtain in particular

$$
\left(\begin{array}{c}
0 \\
p_{1}^{+}\left(\omega_{i}\right) \\
p_{1}^{+}\left(\omega_{j}\right)
\end{array}\right)=p_{0}^{+}\left(\frac{1}{\tau}\right)\left(\begin{array}{c}
p_{1}^{+}(\tau) \\
p_{1}^{+} p_{l}^{-}(\tau) \\
p_{1}^{+} p_{j}^{-}(\tau)
\end{array}\right)+p_{1}^{+}\left(\frac{1}{\tau}\right)\left(\begin{array}{c}
p_{0}^{+}(\tau) \\
p_{0}^{+} p_{1}^{-}(\tau) \\
p_{0}^{+} p_{j}^{-}(\tau)
\end{array}\right)
$$

Now let us assume that $\tau$ is such that the first two column vectors (9.1) are linearly independent. Each vector on the right-hand side of (9.2) is then linearly dependent from the first two column vectors in (9.1) and so is the vector on the left-hand side of (9.3). Thus we conclude that the following three vectors

$$
\left(\begin{array}{c}
\tau \\
p_{i}^{-}(\tau) \\
p_{i}^{-}(\tau)
\end{array}\right),\left(\begin{array}{c}
0 \\
p_{1}^{+}\left(\omega_{i}\right) \\
p_{1}^{+}\left(\omega_{j}\right)
\end{array}\right),\left(\begin{array}{c}
0 \\
p_{n}^{+}\left(\omega_{i}\right) \\
p_{n}^{+}\left(\omega_{j}\right)
\end{array}\right)
$$

are linearly dependent yielding (1.24), (if $\tau \neq 0$ ). 


\section{References}

1 Ohta, Y., Satsuma, J., Takahashi, D., Tokihiro, T.: Prog. Theor. Phys., Suppl. 94, 219 (1988)

2 Date, E., Jimbo, M., Kashiwara, M., Miwa, T.: In: Nonlinear integrable systems - classical quantum theory, ed. Jimbo, M., Miwa, T., Singapore: World Scientific, (1983), p. 39; Jimbo, M., Miwa, T.: Publ.RIMS, Kyoto Univ. 19, 943 (1983)

3 Sato, M., Sato, Y.: In: Nonlinear partial differential equations in applied sciences, ed. Fujita, H., Lax, P.D., Strang, G., Tokyo/Amsterdam: Kinokuniya/North Holland, (1983), p. 259

4 Kaup, D.J.: Prog. Theor. Phys. 54, 396 (1975)

5 Broer, L.J.F.: Appl.Sci. Res. 31, 377 (1975)

6 Kupershmidt, B.A.: Commun. Math. Phys. 99, 51 (1985)

7 Matsukidaira, J., Satsuma, J., Strampp, W.: Phys. Lett. A 147, 467 (1990)

8 Satsuma, J., Matsukidaira, J., Kajiwara, K.: In: Solitons and Chaos, Antoniou, I., Lambert, F.J., (eds.)Berlin, Heidelberg, New York, Springer, (1991), p. 264

9 Hietarinta, J., Kajiwara, K., Matsukidaira, J., Satsuma, J.: In Nonlinear Evolution Equations and Dynamical Systems. Boiti, M., Martina, L., Pempinelli, F., (eds.), Singapore: World Scientific, (1992), p. 30

10 Satsuma, J., Kajiwara,K., Matsukidaira, J., Hietarinta, J.: J. Phys. Soc. Jpn. 9, 3096 (1992)

11 Cheng, Y., Li, Y.S.: Phys. Lett. A 157, 22 1991; J. Phys. A 25, 419 (1992)

12 Konopelchenko, B.G., Strampp, W.: Inverse Problems 7, L17 1991; J. Math. Phys. 33, 3676 (1992)

13 Konopelchenko, B.G., Sidorenko, J., Strampp, W.: Phys. Lett. A 157, 17 (1991)

14 Sidorenko, J., Strampp, W.: Inverse Problems 7, L37 (1991)

15 Xu, B.: Inverse Problems 8, L 13 1992; Inverse Problems 9, 355 (1993)

16 Cheng,Y.: J. Math. Phys. 33, 3774 (1992)

17 Xu, B. Li, Y.: J. Phys. A 25, 2957 (1992)

18 Sidorenko, J., Strampp, W.: J. Math. Phys. 34, 1429 (1993)

19 Oevel, W., Strampp, W.: Constrained KP hierarchy and bi- hamiltonian structures Commun. Math. Phys 157, 51 (1993)

20 Oevel, W.: Physica A 195, 533 (1993)

Communicated by M. Jimbo 
